\title{
Low-Cost Smart Self-Adaptive Humidity Sensors with Parametric Adaptation
}

\author{
Yurish Sergey \\ CDEI, Technical University of Catalonia (UPC-Barcelona) \\ C/Llorens Artigas, 4-6, planta 0, Edifici U, Campus Sud, \\ 08028, Barcelona, Spain \\ Tel.: +34 696067716, fax: +34 93 4011989, e-mail: syurish@sensorsportal.com
}

\begin{abstract}
A low cost humidity and temperature smart self-adaptive sensors systems with parametric adaptation is described in the paper. The system is based on dedicated humidity and temperature transducer HTF3130 and Universal Sensors and Transducers Interface (USTI) integrated circuit, especially designed for such applications. The transducer consists of rugged HTS2030SMD humidity sensor and NTC temperature sensor. The USTI directly converts frequency proportional to the humidity $(10 \% R H \ldots 95 \% R H)$ and resistance proportional to the temperature $\left(-40{ }^{\circ} \mathrm{C} \ldots+85{ }^{\circ} \mathrm{C}\right)$ into digits according to three popular sensor interfaces: RS232, SPI or $I^{2} \mathrm{C}$. Simple decision rule of adaptive measuring algorithm lets chose an appropriate accuracy for frequency-to-digital conversion in particular humidity ranges in order to reduce the conversion time. Sensors system is realized with minimum possible hardware. Experimental results have confirmed high metrological performances of designed sensors system.
\end{abstract}

\section{Introduction}

A relative humidity is one of main parameter in environmental and meteorological measurements, air control in different climatic systems, building or home monitoring, etc. [1]. In addition, humidity measurements are used in different industrial manufacturing processes for paper, food, textile, semiconductors, etc. [2].

Many designed and described smart humidity sensors and sensor systems are based on humidity sensing elements and appropriate readout electronics that generate a voltage output. Then, an analog-todigital converter is used in order to produce a digital output [3-5]. However, the rapid growth of the microelectronics field and availability of inexpensive but high performance Universal Sensors and Transducers Interface (USTI) integrated circuit and humidity, moisture, dew point sensors and sensing modules with frequency output [6-8] have led to an increased interest in the implementation of low cost self-adaptive smart humidity sensors and sensor systems, which use a frequency as an informative parameter of humidity sensor's output.

\section{Humidity Sensor System Design}

The designed sensor system consists of dedicated humidity and temperature transducer HTF3130 [9] and USTI that especially designed for precision frequency-to-digital conversion and signal conditioning in such applications (Fig. 1). The transducer consists of rugged HTS2030SMD humidity sensor and NTC temperature sensor. The frequency on the fist transducer's output proportionals to the relative humidity $(10 \% \mathrm{RH} \quad \ldots \quad 95 \% \mathrm{RH})$, and resistive on the second output - to the temperature $\left(-40{ }^{\circ} \mathrm{C} \ldots+85{ }^{\circ} \mathrm{C}\right)$. Oscillogram at humidity sensor's output at $55 \mathrm{RH} \%$ is shown in Fig. 2. The sensor system can be interfaced to a PC or work autonomously in a master communication mode. The USTI is based on the novel patented modified method of the dependent count [10] and has programmable quantization error (from $1 \%$ to $0.0005 \%$ ), which is constant in all frequency range, scalable resolution, broad frequency measuring range from $0.05 \mathrm{~Hz}$ to $9 \mathrm{MHz}$ withot prescaling and $144 \mathrm{MHz}$ with prescaling, and non-redundant conversion time. It directly converts the frequency and resistance into digitals according to three popular sensor interfaces: RS232, SPI and I ${ }^{2} \mathrm{C}$.

The circuit diagram of humidity and temperature sensors system is shown in Fig. 3 and commands for RS232 communication mode at adaptive measurement of humidity and temperature by the USTI - in Fig.4. Taking into account that maximal resistance of NTC temperature sensor $R_{x}=262960 \mathrm{Ohm}$ [9], the reference calibration resistor $R_{c}$ should be chosen form the range $0<R_{x}<R_{c}$ and is $263 \mathrm{Ohm}$. 


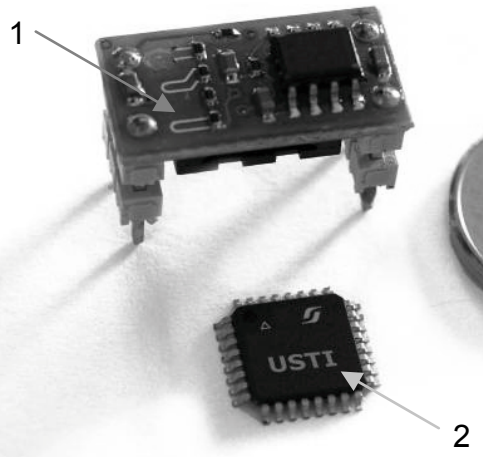

Fig.1. Main components of humidity smart sensor systems: (1) - humidity sensing module with frequency and resistive outputs; (2) - USTI.

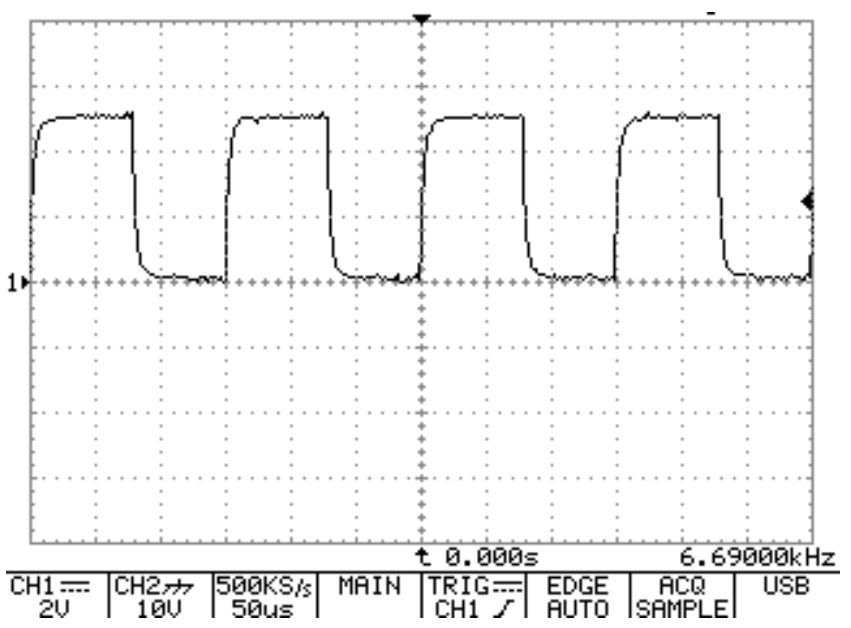

Fig.2. Oscillograms at humidity sensor's output at $55 \% \mathrm{RH}$.

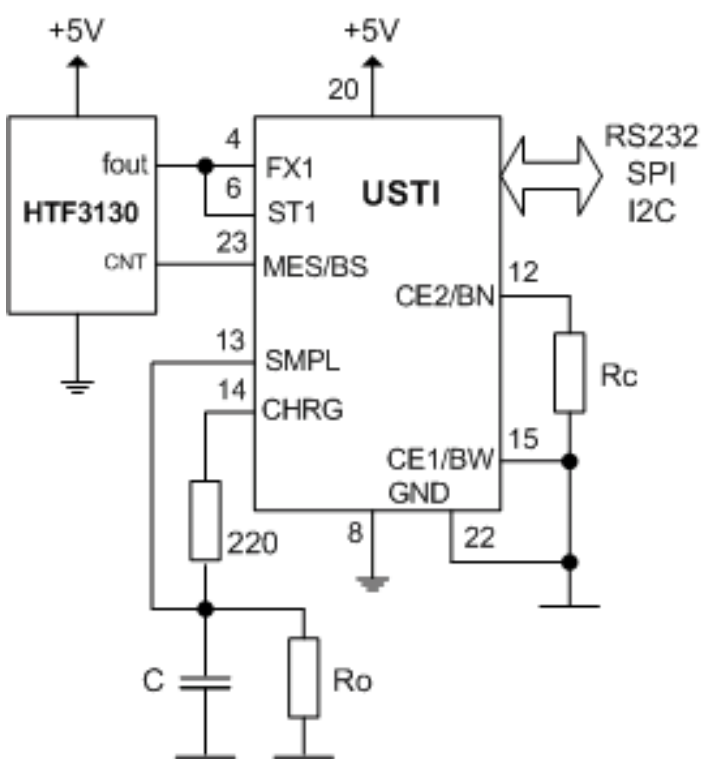

Fig.3. Circuit diagram of humidity and temperature sensors system. 
$>\mathrm{M} 00$; $\quad$ Set a frequency measurement mode in the $1^{\text {st }}$ channel

$>$ A02; $\quad$ Set the relative error $0.25 \%$

$>$ S; $\quad$ Start a frequency measurement (humidity)

$>\mathrm{R} ; \quad$ Read a result of frequency measurement (humidity)

$>$ M10; $\quad$ Set a resistance $R_{x}$ measurement mode

$>$ E263000.0; $\quad$ Set the reference value of $R_{c}=263 \mathrm{k} \Omega$

$>$ W1B; $\quad$ Set the charging time $100 \mathrm{~ms}$

$>\mathrm{S}$; $\quad$ Start a resistive measurement (temperature)

$>\mathrm{R}$; $\quad$ Read a result of resistive measurement (temperature)

;Here microcontroller or computer should check the condition for an algorithm changing and prepare the ;USTI to measure frequency with $0.5 \%$ relative error if a value of humidity is in the $0-10 \% R H$ or ;90-100\% RH relative humidity range.

$>\mathrm{M} 00$; $\quad$ Set a frequency measurement mode in the $1^{\text {st }}$ channel

$>$ A00; $\quad$ Choose the relative error of measurement $1 \%$

$>\mathrm{S}$; $\quad$ Start a frequency measurement (humidity)

$>\mathrm{R} ; \quad$ Read a result of frequency measurement (humidity)

$>$ M10; $\quad$ Set a resistance $R_{x}$ measurement mode

$>$ E263000.0; Set the reference value of $R_{c}=263 \mathrm{kOm}$

$>$ W1B; $\quad$ Set the charging time $100 \mathrm{~ms}$

$>\mathrm{S}$; $\quad$ Start a resistive measurement (temperature)

$>\mathrm{R}$; $\quad$ Read a result of resistive measurement (temperature)

Fig. 4. Commands for RS232 communication mode at adaptive measurement of humidity and temperature by the USTI.

The capacitance $C \geq 0.002 / R_{c}(\mathrm{~F})$ and the time of capacitance charge $T=2200 \times C$ (sec). The values $R_{0}$ and $T$ must be set up with the appropriate commands $E$ and $W$ respectively (Fig.4).

The developed adaptive algorithm for frequency measurements is based on parametrical adaptation It means a possibility to change accuracy on conversion speed and conversely. The choice of variants is made according to the current result of measurement $\beta$. So, for the modified method of the dependent count the equations of measurements are the following:

$$
\left\{\begin{array}{l}
\lambda^{*}{ }_{j}=T_{s} L \gamma_{j}(t), \text { if } F_{x}\left(\beta^{*}\right) \in I_{f} \\
\lambda^{*}{ }_{j}=\delta_{s} L \gamma_{j}(t), \text { if } F_{x}\left(\beta^{*}\right) \notin I_{f}
\end{array} \text { at } I_{f} \in I,\right.
$$

where $F_{x}\left(\beta^{*}\right)$ is the characteristic of input action or measuring conditions, which value defines the decision for change of measuring algorithm (parameters of system); $I_{f}$ is the subset of certain area $I$ of possible values of characteristic $F_{x}\left(\beta^{*}\right)$, the set membership to which defines the necessity to change of system's parameters. Generally, the task of determination of area $l_{f}$ for the resulted parameter arises.

In the smart humidity sensors system such adaptation can be used by the following way. Various frequency output humidity sensors have different errors in various parts of measuring range. For example, the humidity module HFT3130 (Humirel) has the absolute error, which is changed in different part of humidity range $(0$ to $99 \% \mathrm{RH})$ in 1.67 times [9]. Based on sensors systems design considerations, in order to be neglected, the relative error of frequency-to-digital conversion should be chosen in one order (or at the least in 5 times) less than the relative error of sensor. Based on the parametric adaptation, it is possible to reduce the conversion time in self-adaptive smart humidity sensor systems. In this case, the programmable relative error for frequency-to-digital conversion must be changed dependent on the current measuring range of humidity and chosen $0.5 \%$ for $0 \ldots 10 \% \mathrm{RH}$ and $90 \ldots 100 \% \mathrm{RH}$ (subset $I_{f}$ of $I$ set of all possible values), and $0.25 \%$ for the rest of humidity range. This will reduce conversion time on $0.5 \mathrm{~ms}$. Obviously, if the range of sensor's error is changing in wide limits, the effect of measurement time reduction will be greater. 


\section{Experimental Results}

Humidity sensor system was investigated experimentally in the climatic chamber CTS T-40/60 at $+25{ }^{0} \mathrm{C}$ (Fig. 5). Experimental results are shown in Fig. 6. Statistical characteristics at frequency measurements (55 RH \%) and density of distribution are shown in Table 1 and Fig.7 The relative error of frequency measurement by the USTI was not more than $0.0077 \%$ in all frequency range from 6200 to $7155 \mathrm{~Hz}$.

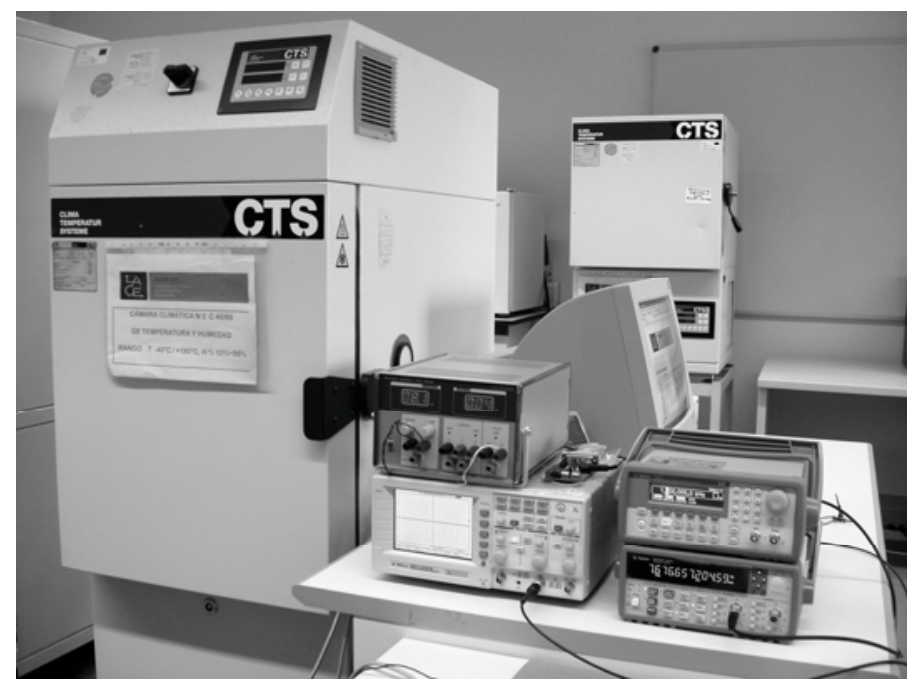

Fig.5. Climatic chamber CTS 40/60 and measuring equipment.

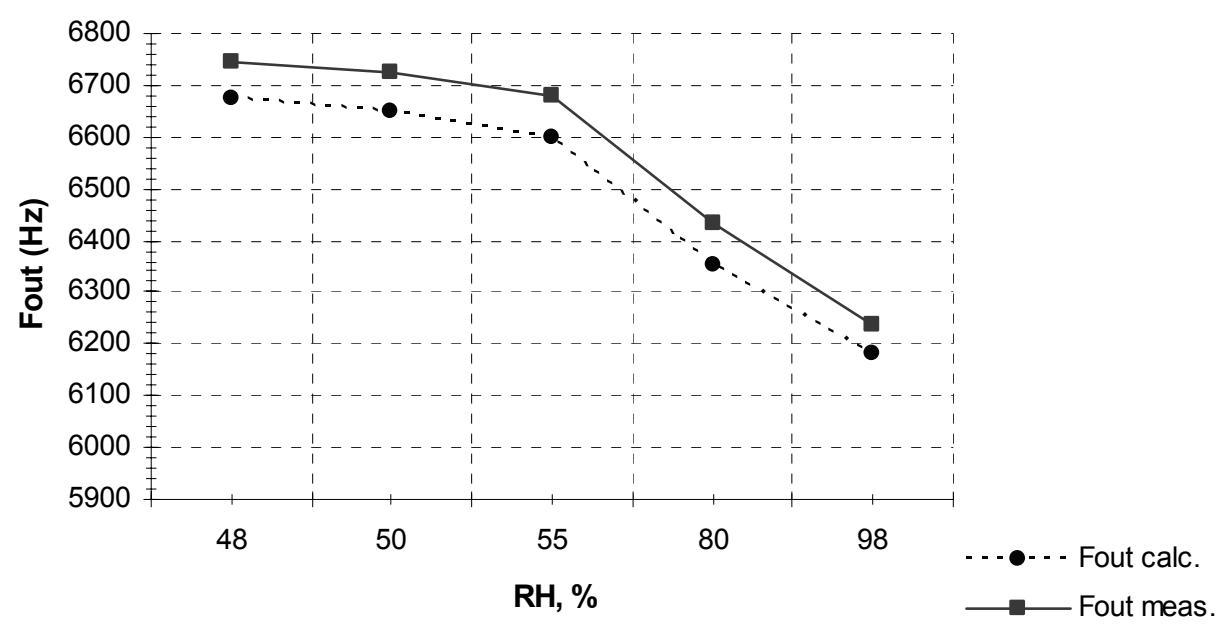

a)

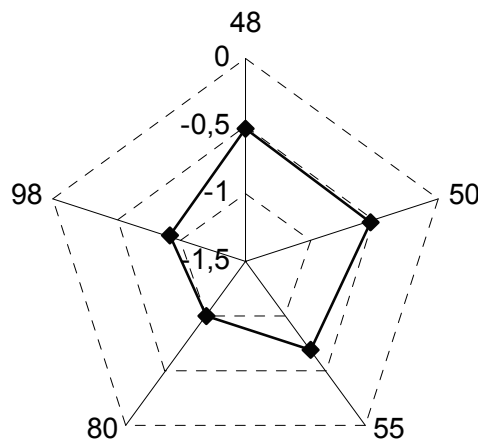

b)

Fig.6. Dependences of output frequency (a) and absolute error (b) on relative humidity $\mathrm{RH}, \%$. 
Table 1. Statistical characteristics at $f_{x}$ measurements (55 RH \%).

\begin{tabular}{|l|l|}
\hline \multicolumn{1}{|c|}{ Parameter } & \\
\hline \hline Number of measurements, $N$ & 60 \\
\hline Minimum $f_{x \min }, \mathrm{Hz}$ & 6678.0817 \\
\hline Maximum $f_{x \max }, \mathrm{Hz}$ & 6683.5299 \\
\hline Sampling Range, $f_{x \max }-f_{x \min }, \mathrm{Hz}$ & 5.4481 \\
\hline Median & 0 \\
\hline Arithmetic Mean, $\mathrm{Hz}$ & 6681.2234 \\
\hline Variance, $\mathrm{Hz}$ & 3.3891 \\
\hline Standard Deviation, $\mathrm{Hz}$ & 1.8409 \\
\hline Coefficient of Variation & 3629.2505 \\
\hline Confidence Interval at probability $P=97 \%$ & $6680.7076 \leq f_{x} \leq 6681.7391$ \\
\hline Relative error, $\%$ & $0.0077<0.01$ \\
\hline$\chi^{2}-$ test $(S)$ at: $k=5 ; P=97 \% \chi^{2} \max =10.9$ & 10.6667 \\
\hline Hypothesis about uniform distribution & $S<\chi_{\text {max }}^{2}$ (accepted) \\
\hline
\end{tabular}

$k$ is the number of equidistant classes;

$S$ is the sum of deviations between the data set and the assumed distribution;

$\chi_{\max }^{2}$ is the maximal possible allowable argument of the $\chi^{2}$ distribution.

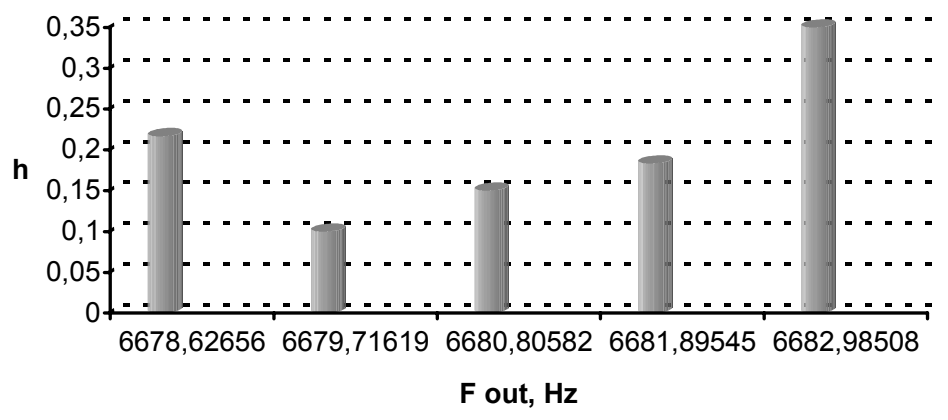

Fig.7. Density of distribution for frequency output measuring results at $55 \% \mathrm{RH}$.

The difference between calculated humidity and measured valuse is due to a difference in the temperature operating range (Fig.6 a). The typical response look-up table data were obtained according to the polynomial reference curve for modeled signal output $\left(F_{\text {out }}=7314-16.79 \times \mathrm{RH}+0.0886 \times \mathrm{RH}^{2}-\right.$ $-0.000358 \times \mathrm{RH}^{3}$ ) at $+25^{\circ} \mathrm{C}[9]$. The real experiments were carry out at $+25^{\circ} \mathrm{C} \pm 0.5^{\circ} \mathrm{C}$.

The $\chi^{2}$-test for goodness of fit was applied to investigate the significance of the differences between observed data in the histograms and the theoretical frequency distribution for data from a uniform population. Taking into account that the sum of deviations between the data set and the assumed distribution $S<\chi^{2}$, the hypothesis about uniform distribution can be accepted.

Taking into account that the USTI has two independent channels, it is possible instead of resistive NTC or RTD temperature sensors to use any quasi-digital temperature sensor with frequency, priod, duty-cycle or PWM output [11]. The temperature sensor can be used in such sensor system for signal drift compensation. The quasi-digital temperature sensors also have different absolure errors in different temperature ranges, hence, the seff-adaptive algorithms aslo can be used in order to reduce the comversion time.

A capasitance-type humidity sensing element aslo can be connected directly to the USTI because of IC can convert a capacitance-to-digital. In this case one external resistor and reference capasitor should be used.

Due to high metrological performance of the USTI this IC is also suitable for interfacing of any humidity sensors based on quartz crystal microbalance (QCM) gravimetric devices, for example, described in [6-7]. 
Another smart function in the designed humidity sen-sor system is a possibility to store in a flash memory of USTI a TEDS according to the IEEE 1451.4 standard with the aim of self-identification.

\section{Conclusions}

The designed low-cost humidity and temperature sensors system has demonstrated high metrological performance, efficiency and reliability. It is based on minimal possible hardware and has intelligent functions as self-adaptation and self-identification. It can contain any quasi-digital humidity sensors with frequency, period, duty-cycle and PWM or capacitance output, quasi-digital temperature or resistive output sensors and Universal Sensors and Transducers Interface (USTI). The device is based on a novel method for frequency measurements and signal conditioning concept. Further research and development aims towards creation multisensor systems for different applications.

\section{Aknowledgements}

This research and development was funded in the frame of EC Marie Curie Excellence Chairs (EXC) project MEXT-CT-2005-023991 Smart Sensors Systems Design (SMARTSES). The author would also like to thank Dr. Elena Krotenko from LACE (Barcelona) for her assistance in experiments in climatic chamber.

\section{References}

[1]. Matos, R., Cabral, S., Bravo, N., Cordeiro, A., Palma, J., Digital Temperature and Humidity Monitoring Unit for Re-mote Applications, in Proceedings of IEEE International Symposium on Consumer Electronic, (ISCE'2008), 14-16 April 2008, pp.1-4.

[2].Hideo Shibata, Masahiro Ito, Masahiro Asakursa, Kenzo Watanabe, A Digital Hygrometer Using a Polyimide Film Relative Humidity Sensor, IEEE Transactions on Instrumentation and Measurement, Vol. 45, No. 2, April 1996, pp.564-569.

[3].Sung P. Lee, Ji G. Lee, Joong W. Chang, Joo N. Kim , Sang H. Lee, Shaestagir Chowdhury, Integrated Sensor System for Humidity Sensing of Robots Using Analog Mixed CMOS Technology, in Proceedings of IEEE International Workshop on Robotic and Sensors Environments (ROSE 2007), Ottawa, Canada, 12-13 October 2007, pp.1-4.

[4].Giovanni Bucci, Marco Faccio, Carmine Landi, The Implementation of a Smart Sensor Based on Piece-Linear A/D Converter, in Proceedings of IEEE Instrumentation and Measurement Technology Conference, Ottawa, Canada, May 19-21, 1997, pp. 1173-1177.

[5].Xiaojing Shi, Hiroki Matsumoto, Kenji Murao, A High-Accuracy Digital Readout Technique for Humidity Sensor, IEEE Transactions on Instrumentation and Measurement, Vol.50, No.5, October 2001, pp. 1277-1282.

[6].Galatsis, K., Wenmin Qu, Wlodarski W., Quartz Crystal Microbalance Humidity Sensor with Porous Electrodes, in Proceedings of Conference on Optoelectronic and Microelectronic Materials Devices, 1999 , pp. $373-375$.

[7].H. Ito, S. Kakuma, R. Ohba, K. Nod, Development of a Humidity Sensor using Quartz Crystal Microbalance, in Proceedings of Annual Conference SICE, Fukui, Japan, 4-6 August 2003, pp.1175-1178.

[8].List of Humidity and Moisture Sensors Manufacturers at Sensors Web Portal (www.sensorsportal.com) http://www.sensorsportal.com/HTML/SENSORS/HumiditySens_Manufacturers.htm

[9].Temperature and Humidity Module HTF3130, Humirel Sensor Device Data, HPC050V0, 2004.

[10].Yurish, S., Novel modified method of the dependent count for high precision and fast measurements of frequency-time parameters of electric signals, in Proceedings of 2008 IEEE International Instrumentation \& Measurement Technology Conference (I $\left.{ }^{2} \mathrm{MTC}\right)$, Victoria, Vancouver Island, British Columbia, Canada, 12-15 May 2008, pp 876-881.

[11].List of Temperature Sensors Manufacturers at Sensors Web Portal (http://www.sensorsportal.com): http://www.sensorsportal.com/HTML/SENSORS/TempSens_Manufacturers.htm 